\title{
High-Level Expression of Various Apolipoprotein(a) Isoforms by "Transferrinfection": The Role of Kringle IV Sequences in the Extracellular Association with Low-Density Lipoprotein ${ }^{\dagger}$
}

\author{
Sasa Frank, ${ }^{\ddagger}$ Krisztina Krasznai, ${ }^{\S}$ Srdan Durovic, ${ }^{\ddagger}$ Eva-Maria Lobentanz, ${ }^{8}$ Hans Dieplinger, ${ }^{8}$ Ernst Wagner,"l \\ Kurt Zatloukal," Matt Cotten," Gerd Utermann, ${ }^{\S}$ Gert M. Kostner, ${ }^{\ddagger}$ and Rudolf Zechner ${ }^{*}, \downarrow$ \\ Institute of Medical Biochemistry, Karl-Franzens University, Graz, Austria, Institute of Medical Biology and Human Genetics, \\ University of Innsbruck, Innsbruck, Austria, and Institute of Molecular Pathology, University of Vienna, Vienna, Austria
}

Received February 14, 1994; Revised Manuscript Received June 20, $1994^{\circ}$

\begin{abstract}
Characterization of the assembly of lipoprotein(a) [Lp(a)] is of fundamental importance to understanding the biosynthesis and metabolism of this atherogenic lipoprotein. Since no established cell lines exist that express Lp(a) or apolipoprotein(a) [apo(a)], a "transferrinfection" system for apo(a) was developed utilizing adenovirus receptor- and transferrin receptor-mediated DNA uptake into cells. Using this method, different apo(a) cDNA constructions of variable length, due to the presence of 3, 5, 7, 9, 15, or 18 internal kringle IV sequences, were expressed in cos- 7 cells or $\mathrm{CHO}$ cells. All constructions contained kringle IV-36, which includes the only unpaired cysteine residue (Cys-4057) in apo(a). r-Apo(a) was synthesized as a precursor and secreted as mature apolipoprotein into the medium. When medium containing r-apo(a) with 9,15 , or 18 kringle IV repeats was mixed with normal human plasma LDL, stable complexes formed that had a bouyant density typical of $L p(a)$. Association was substantially decreased if Cys-4057 on r-apo(a) was replaced by Arg by site-directed mutagenesis or if Cys-4057 was chemically modified. Lack of association was also observed with $\mathrm{r}$-apo(a) containing only 3,5 , or 7 kringle IV repeats without "unique kringle IV sequences", although Cys- 4057 was present in all of these constructions. Synthesis and secretion of $\mathrm{r}$-apo(a) was not dependent on its sialic acid content. r-Apo(a) was expressed even more efficiently in sialylation-defective $\mathrm{CHO}$ cells than in wild-type $\mathrm{CHO}$ cells. In transfected $\mathrm{CHO}$ cells defective in the addition of $N$-acetylglucosamine, apo(a) secretion was found to be decreased by $50 \%$. Extracellular association with LDL was not affected by the carbohydrate moiety of $r$-apo(a), indicating a protein-protein interaction between $\mathrm{r}$-apo(a) and apoB. These results show that, besides kringle IV-36, other kringle IV sequences are necessary for the extracellular association of r-apo(a) with LDL. Changes in the carbohydrate moiety of apo(a), however, do not affect complex formation.
\end{abstract}

Lipoprotein(a) $[\mathrm{Lp}(\mathrm{a})]^{1}$ is currently the subject of intensive investigation because elevated plasma concentrations of $\mathrm{Lp}$ (a) represent an independent risk factor for myocardial infarction and stroke (Scanu \& Fless, 1990; Utermann, 1989, 1990; Edelberg \& Pizzo, 1991). Despite its clinical importance, the physiological function of $\mathrm{Lp}(\mathrm{a})$ is unknown. Structurally, $L p(a)$ resembles a low-density lipoprotein (LDL)

† This work was supported by the Austrian Science Foundation, Grants S4601 (G.M.K.), S4602 (G.M.K., G.U.), S4603, S7110 (R.Z.), S4604 (H.D.), and S4610 (G.U.).

* To whom correspondence should be addressed at the Institute of Medical Biochemistry, Karl-Franzens University, Graz, Harrachgasse 21, A-8010 Graz, Austria.

$\ddagger$ Karl-Franzens University, Graz.

8 University of Innsbruck.

II University of Vienna.

- Abstract published in Advance ACS Abstracts, September 15, 1994

${ }_{1}^{1}$ Abbreviations: A po, a polipoprotein; Lp, lipoprotein; r-, recombinant; CHO, Chinese hamster ovary; LDL, low-density lipoprotein; HDL, highdensity lipoprotein; VLDL, very low density lipoprotein; SDS, sodium dodecyl sulfate; PAGE, polyacrylamide gel electrophoresis; UTR, untranslated region; DTT, dithiothreitol; FCS, fetal calf serum; LPDS lipoprotein-deficient serum; DMEM, Dulbecco's modified essential medium; ECL, enhanced chemiluminescence; HBS, HEPES-buffered saline [ $20 \mathrm{mM} N$-(2-hydroxyethyl)piperazine- $N^{\prime}-2$-ethanesulfonic acid, pH 7.3, and $150 \mathrm{mM} \mathrm{NaCl}$; PBS, $0.15 \mathrm{M} \mathrm{NaCl}, 2.7 \mathrm{mM} \mathrm{KCl}, 5.4 \mathrm{mM}$ $\mathrm{Na}_{2} \mathrm{HPO}_{4} \cdot 7 \mathrm{H}_{2} \mathrm{O}, 1.5 \mathrm{mM} \mathrm{KH}_{2} \mathrm{PO}_{4}, \mathrm{pH} 7.4 ; \mathrm{TBS}, 0.15 \mathrm{M} \mathrm{NaCl}, 10 \mathrm{mM}$ Tris- $\mathrm{HCl}$, pH 7.4; PMSF, phenylmethanesulfonyl fluoride; EDTA, ethylenediaminetetraacetic acid; EGTA, ethylene glycol bis ( $\beta$-aminoethyl ether)- $N, N, N^{\prime}, N^{\prime}$-tetraacetic acid; ELISA, enzyme-linked immunosorbent assay: DELFIA, dissociation-enhanced lanthanide fluorescence immunoassay. particle with apolipoprotein (apo) B-100 and apo(a) as major structural protein constituents. Biochemical evidence suggests a direct linkage of apo(a) to apoB by a disulfide bridge (Sommer et al., 1991; Guevara et al., 1993). A single free cysteine residue on apo(a) corresponding to Cys-4057 on the published apo(a) cDNA sequence is believed to be involved in this heterodimer formation (McLean et al., 1987). Apo(a) is a glycoprotein with a high sialic acid content that shares extensive protein sequence homology with plasminogen (McLean et al., 1987). This homology includes the presence of sequences similar to kringle IV, kringle $\mathrm{V}$, and the protease domain of plasminogen on apo(a). The genes for plasminogen and apo(a), together with two apo(a) pseudogenes, are located within a gene cluster on chromosome 6 (Ichinose, 1992; Malgaretti et al., 1992). At least 30 apo(a) alleles exist in humans, which determine proteins of variable size (Lackner et al., 1991; Kamboh et al., 1991). These size differences are due to variation in the number of kringle IV repeats on apo(a) (Kraft et al., 1992). In caucasians, as well as in the cynomologous monkey model, Lp(a) plasma concentrations are known to be partially determined by the number of kringle IV repeats on the apo(a) gene (Kraft et al., 1992; Boerwinkle et al., 1992; Utermann et al., 1990; Koschinsky et al., 1990; Azrolan et al., 1991).

Apo(a) and apoB are both produced in the liver. Metabolic studies in humans have demonstrated that triglyceride-rich lipoproteins, which are well-established precursors of LDL, do not represent metabolic precursors for Lp(a) (Krempler et al., 1979; Sandholzer et al., 1992). The question of whether 
apo(a) forms a complex with apoB in hepatocytes or whether apo(a) binds to LDL only after its secretion to the interstitium or the plasma compartment remains open. Evidence has been found that free apo(a) can be secreted from liver cells. Free apo(a) is detectable in the plasma of patients suffering from abetalipoproteinemia (Menzel et al., 1990). In transgenic mice that overexpress human apo(a), the apolipoprotein is found at high concentrations in the plasma of the animals, despite its inability to associate with mouse LDL to form Lp(a) (Chiesa et al., 1992). Finally, when r-apo(a) was expressed in transfected Hep G2 cells a large amount of r-apo(a) was found in its free, non-apoB-associated form (Koschinsky et al., 1991). Evidence supporting an extracellular association between apo(a) and LDL was obtained when the injection of human LDL into transgenic mice expressing human apo(a) resulted in the rapid and efficient formation of $\mathrm{r}$-apo(a)/LDL complexes which were resistant to SDS and strongly resembled Lp(a) (Chiesa et al., 1992). Lp(a)-like particles were also found in transgenic mice expressing human apo(a) together with human apoB (Linton et al., 1993). Finally, several authors reported a strong binding affinity of recombinant apo(a) [r-apo(a)] or apo(a) produced in primary baboon liver cells to LDL in vitro (White et al., 1993; Trieu, 1991; Phillips et al., 1993; Koschinsky et al., 1993). Two recent studies utilizing site-directed mutagenesis demonstrated the direct involvement of Cys-4057 in the association process (Koschinsky et al., 1993; Brunner et al., 1993).

Here we report the development of a system for the efficient expression of free $\mathrm{r}$-apo(a) in cos-7 cells and different $\mathrm{CHO}$ cell lines. Using this system it was possible to investigate the extracellular association of $r$-apo(a) of varying sizes with $L D L$, the function of the carbohydrate moiety of $r-a p o(a)$ in this process, and the consequences of modifications of Cys-4057 for the extracellular binding of r-apo(a) to LDL.

\section{EXPERIMENTAL PROCEDURES}

Materials and Cell Lines. DMEM (Dulbecco's minimum essential medium) was obtained from Boehringer Mannheim (Germany), and culture dishes were from Nunc (Roskilde, Denmark). All cells lines used were obtained from the American Type Culture Collection. Cos-7 are SV40 transformed African green monkey kidney cells (ATCC CRL 1651). CHO (Chinese hamster ovary) cell lines included a wild-type cell line (Pro-5-, ATCC CRL 1781), a line with blocked N-linked glycosylation (Lec 1, ATCC CRL 1735), a cell line with drastically decreased protein sialylation (Lec 2, ATCC CRL 1736), and a cell line with UDP-galactose deficiency in the Golgi apparatus (Lec 8, ATCC CRL 1737). Anti-apo(a) and anti-apoB antibodies were produced in our own la boratories as described previously (Kraft et al., 1988). Horseradish peroxidase-linked protein $\mathbf{A}$ and an enhanced chemiluminscent detection kit (ECL kit) were obtained from Amersham Corp. Standard solutions for phenotyping apo(a) isoforms were purchased from Immuno AG (Vienna, Austria). Monensin was from Sigma (St. Louis, MO). Replicationdefective biotinylated adenovirus ( $\mathrm{dI} 312$ ) in $40 \%$ glycerol, streptavidin-polylysine conjugate and human transferrinpolylysine conjugate in HBS buffer (20 mM HEPES, $\mathrm{pH} 7.3$, and $150 \mathrm{mM} \mathrm{NaCl}$ ) were produced at the Institute of Molecular Pathology, Vienna (Wagner et al., 1990).

Construction of Apo(a) Expression Vectors. Apo(a) expression plasmids containing DNA sequences coding for 3 , $5,7,9,15$, and 18 kringle IV-like domains, as well as the kringle V-like and protease-like domains, were assembled using several apo(a) cDNA clones reported by McLean et al. (1987). Standard recombinant DNA techniques were followed (Sam- brook et al., 1989). Specific apo(a) cDNA fragments (see below) were ligated into the EcoRI site of pSG 5. This expression vector contains the simian virus (SV 40) early promoter, rabbit $\beta$-globin intron II, and the SV 40 polyadenylation signal (Green et al., 1988). For transfection experiments, plasmid DNA was isolated by the alkaline lysis method and purified by cesium chloride density gradient ultracentrifugation.

pSG5-S contained three kringle IV-like repeats. A 298-bp EcoRI-Bam HI fragment isolated from $\lambda 18$ cDNA (McLean et al., 1987) encoding the 5'-untranslated region, signal sequence, and 216 bp of kringle IV-1 repeat of apo(a) was fused to a 2024-bp BamHI-EcoRI fragment isolated from $\lambda 41$ cDNA (McLean et al., 1987) encompassing the last 117 bp of kringle IV-35, kringles IV-36 and IV-37, kringle V, protease domain, and $67 \mathrm{bp}$ of $3^{\prime}$-untranslated sequence.

pSG5-M contained nine kringle IV repeats. A 709-bp EcoRI-Hhal fragment from $\lambda 18$ encoding the 5 '-untranslated region, signal sequence, kringle IV-1, and $294 \mathrm{bp}$ of kringle IV-2 was fused to a 3641 -bp $H h a I-E c o$ R I fragment from $\lambda 41$ containing the last 48 bp of kringle IV-30, kringles IV-31 through IV-37, kringle V, protease domain, and $67 \mathrm{bp}$ of 3'untranslated sequence.

pSG5-L contained 15 kringle IV repeats. A 1735-bp EcoRI-HhaI fragment from $\lambda 18$ encoding the 5 '- untranslated region, signal sequence, kringle IV repeats $1-4$, and 299 bp of kringle IV-5 was fused to a 4669-bp fragment from $\lambda 41$ encompassing the last 48 bp of kringle IV-27, kringles IV-28 through IV-37, kringle V, protease domain, and 67 bp of 3'untranslated region.

pSG5-XL contained 18 kringle IV repeats. A 2077-bp EcoRI-HhaI fragment from $\lambda 18$, encoding the $5^{\prime}$-untranslated region, signal sequence, kringle IV repeats $1-5$, and $294 \mathrm{bp}$ of kringle IV- 6 was fused to a 5353-bp fragment from pSG5-L encompassing the last 48 bp of kringle IV-3, kringle IV-4, 294 bp of kringle IV-5, the last 48 bp of kringle IV-27, kringle IV repeats $28-37$, kringle $V$, protease domain, and 67 bp of 3'untranslated region.

pSG5-Arg is identical to pSG5-XL except that the unique free cysteine residue (Cys 4057) was replaced by Arg using site-directed mutagenesis. A 2.2-kb KpnI-SspI fragment was cloned into $\mathrm{M} 13 \mathrm{mp} 19$ and mutagenesis was performed with single-stranded DNA and a mutant oligonucleotide using the in vitro mutagenesis kit 2.1 from Amersham. After the mutagenesis experiment, sequence analysis confirmed that the $T$ to $C$ base change was successfully introduced. The mutated DNA fragment was recloned into pSG5-XL.

pSG5-3.0 contained five kringle IV repeats. A 982-bp EcoRI-BamHI fragment from $\lambda 18$ encoding the 5 '-untranslated region, signal sequence, kringle IV-1, kringle IV-2, and 225 bp of kringle IV-3 was fused to a 2024-bp BamHI-EcoRI fragment isolated from $\lambda 41$ encompassing the last $117 \mathrm{bp}$ of kringle IV-35, kringle IV-36, kringle IV-37, kringle V, protease domain, and $67 \mathrm{bp}$ of $3^{\prime}$-untranslated region.

pSG5-3.6 contained seven kringle IV repeats. A 1963-bp EcoRI-PstI fragment was isolated from $\lambda 18$ encoding the 5 -untranslated region, signal sequence, kringle IV repeats $1-5$, and 184 bp of kringle IV- 6 was fused to a $1726-\mathrm{bp}$ Pst I$E c o$ RI fragment from $\lambda 41$ encompassing the last $158 \mathrm{bp}$ of kringle IV-36, kringle IV-37, kringle V, protease domain, and $67 \mathrm{bp}$ of 3'-untranslated region.

Cell Culture and Transfection of Cultured cos-7 and CHO Cells. Cos-7 cells were cultured in DMEM, supplemented with $10 \%$ fetal calf serum (FCS) or 10\% lipoprotein-depleted human serum (LPDS). CHO cells (Pro 5-) and glycosylationdefective CHO cell lines ( Lec 1, Lec 2, and Lec 8) were cultured 
in $\alpha$-MEM supplemented with $10 \%$ FCS or LPDS. Cos-7 cells were plated $24 \mathrm{~h}$ and $\mathrm{CHO}$ cells $48 \mathrm{~h}$ prior to DNA transfection in $\mathrm{P} 60$ dishes. Cell density reached approximately 300000 cells/dish at the time of transfection.

To obtain a high efficiency of transfection with the large plasmids, cells were transfected by a receptor-mediated gene delivery system (Zatloukal et al., 1992; Cotten et al., 1992). A streptavidin-polylysine conjugate was diluted in HBS buffer ( $20 \mathrm{mM}$ HEPES, pH 7.3 , and $150 \mathrm{mM} \mathrm{NaCl}$ ) to a total volume of $100 \mu \mathrm{L}$ and mixed with biotinylated adenovirus diluted in $100 \mu \mathrm{L}$ of HBS buffer. The mixture was incubated for 30 min at room temperature and mixed with $6 \mu \mathrm{g}$ of DNA in $150 \mu \mathrm{L}$ of HBS. After $30 \mathrm{~min}$ at room temperature, the transferrin-polylysine conjugate in $150 \mu \mathrm{L}$ of $\mathrm{HBS}$ was added and incubated again for $30 \mathrm{~min}$ at room temperature. The total mix of $500 \mu \mathrm{L}$ was added to $1.5 \mathrm{~mL}$ of cell medium supplemented with $2 \%$ FCS or LPDS and added to preconfluent cells. After $4 \mathrm{~h}$ the transfection mixture was replaced by fresh DMEM supplemented with $10 \%$ FCS or $10 \%$ LPDS. The amounts of streptavidin-polylysine and transferrinpolylysine conjugate depended strongly on the adenovirus preparation and was optimized for each virus batch used in transfection experiments. For optimization of transfection condition and for normalization of transfection efficiencies, expression plasmids for $\beta$-galactosidase (McGregor \& Caskey, 1989 ) and human growth hormone (Selden, 1987) were cotransfected by the same method in some experiments. $\beta$-Galactosidase assays were performed according to standard techniques (Sambrook et al., 1989). The radioimmunoassay for human growth hormone was purchased from BSM Diagnostics (Vienna, Austria) and the protocol of the manufacturer was followed.

Inhibition of Glycosylation in the Golgi Complex of Transfected Cos-7 Cells. To investigate glycosylation within the Golgi compartment, transfected cos-7 cells were treated with monensin, an inhibitor of protein transport within the Golgi complex (Griffiths et al., 1983). Monensin $(1 \mu \mathrm{M})$ was added to the cell medium and cells were cultivated for an additional $12 \mathrm{~h}$. After incubation, the cell medium was collected, cells were harvested, and cell lysates were prepared as described below.

Recombinant Apo(a) Analysis in Cell Media and Cell Lysates. At various times following transfection, cell medium and cell lysates were analyzed for the presence of $r$-apo(a). Once the medium was aspirated, a mixture of proteinase inhibitors was added to avoid r-apo(a) degradation $(0.2 \mu \mathrm{M}$ aprotinin, $50 \mu \mathrm{M}$ leupeptin, and $1 \mu \mathrm{M}$ PMSF). Free-floating cells were removed by centrifugation for $10 \mathrm{~min}$ at $2000 \mathrm{~g}$ and samples were immediately analyzed or stored at $-70^{\circ} \mathrm{C}$. For the analysis of intracellular r-apo(a), cell lysates were prepared. Cells were washed twice with ice-cold phosphate-buffered saline (PBS), scraped off the culture dishes using a rubber policeman and $1 \mathrm{~mL}$ of lysis buffer ( $1 \%$ Triton X-100, $50 \mathrm{mM}$ HEPES, $\mathrm{pH} 7.2,150 \mathrm{mM} \mathrm{NaCl}, 0.5 \mathrm{mM}$ EDTA, and 0.5 mM EGTA), and transferred to a centrifuge tube. After incubation for $30 \mathrm{~min}$ on ice, cellular debris was removed by centrifugation at $13000 \mathrm{~g}$ for $2 \mathrm{~h}$. Supernatants were further analyzed. For r-apo(a) analysis, cell medium and lysates were diluted (1:1 ratio) with SDS electrophoresis running buffer $(0.1 \%$ bromophenol blue, $1 \%$ glycerol, and $2 \%$ SDS) in the presence (reducing conditions) or absence (nonreducing conditions) of $5 \% \beta$-mercaptoethanol. Samples were heated for $5 \mathrm{~min}$ at $100^{\circ} \mathrm{C}$, cooled down to room temperature, and subjected to SDS-agarose gel electrophoresis or SDS-PAGE. SDS-agarose gel electrophoresis was performed according to the method described by Kamboh et al. (1991). Briefly, the gel contained $1.5 \%$ ultrapure agarose (Gibco Ltd., England) in $90 \mathrm{mM}$ Tris-borate buffer, $\mathrm{pH} 7.4,2 \mathrm{mM}$ EDTA, and $0.1 \%$ SDS. Electrophoresis was carried out in a submarine agarose gel unit using $45 \mathrm{mM}$ Tris-borate buffer, $\mathrm{pH} 7.4$, $0.1 \%$ SDS, and $2 \mathrm{mM}$ EDTA as running buffer. After electrophoresis, proteins were transferred to a nitrocellulose membrane by electroblotting using an LKB 2005 transport power supply with a 2219 thermostatic circulator overnight at $4^{\circ} \mathrm{C}$ in $10 \mathrm{mM}$ Tris and $40 \mathrm{mM}$ glycine buffer, $\mathrm{pH} 7.4$. SDS-PAGE of r-apo(a) containing samples and subsequent immunoblotting was performed essentially as described earlier (Utermann et al., 1987). Briefly, samples were heated for 3 min at $95{ }^{\circ} \mathrm{C}$ in a solution of $5 \%$ SDS and $0.02 \mathrm{mM}$ ethylmorpholine, $\mathrm{pH} 8.6$, in the presence (reducing conditions) or absence (nonreducing conditions) of $5 \% \beta$-mercaptoethanol. A 5- $\mu \mathrm{L}$ aliquot was applied to a $6.6 \%$ polyacrylamide gel and run in a $10 \times 10-\mathrm{cm}$ electrophoresis chamber from Biometra (Göttingen, Germany).

Immunodetection of $r$-Apo(a). After protein transfer, the nitrocellulose membrane was incubated for $60 \mathrm{~min}$ in a solution of 5\% powdered skim milk in Tris-buffered saline, $\mathrm{pH} 7.4$ (TBS), to block the remaining unspecific protein-binding sites. The membrane was then incubated for $3 \mathrm{~h}$ with a rabbit anti human apo(a) (K202, K271) or anti-apoB antiserum at a dilution of 1:1000, washed extensively in TBS containing $0.05 \%$ Tween 20 , and incubated with horseradish peroxidase-labeled protein $A$ as a second antibody for $3 \mathrm{~h}$. After repetitive washing (see above), the membrane was incubated with the ECL Western blotting detection reagent for $1 \mathrm{~min}$ and subjected to autoradiography. Immunoblotting after SDS-PAGE was performed identically, except that in some experiments a monoclonal apo(a) antibody (clone 1A2) was used for detection (Kraft et al., 1988). In that case a goat anti-mouse immunoglobin conjugated with horseradish peroxidase was used as second antibody. Identical conditions were used when a monoclonal antibody specific for kringle $V$ sequences of apo(a) was employed for immunodetection (Huby et al., 1994).

Immunoquantitation of $r$-Apo(a) and Apo B. r-Apo(a) quantitation in cell culture media and fractions thereof was performed with a double-antibody ELISA as described earlier (Menzel et al., 1990). Affinity-purified polyclonal anti-apo(a) antibody was used for coating the ELISA plates, and the horseradish peroxidase-labeled monoclonal antibody $1 \mathrm{~A} 2$ was used for detection (Selden, 1987). This antibody does not cross-react with plasminogen. A commercially available Lp(a) standard from Immuno AG (Vienna, Austria) served as a control. Concentrations of apoB in cell media and ultracentrifugation density gradient fractions were measured by a recently described double-antibody ELISA (Dieplinger et al., 1993). An affinity-purified rabbit polyclonal antibody against human apoB was used for coating. The same antibody was horseradish peroxidase-labeled and used as the detection antibody for apoB in samples and standard. A calibrated standard in a dilution series from 1:700 to 1:240.000 served as secondary standard (calibration serum apolipoprotein, Boehringer Mannheim, Germany).

Association of $r-A p o(a)$ with Human LDL. LDL was prepared by ultracentrifugation in a density range from 1.020 to $1.63 \mathrm{~g} / \mathrm{mL}$ from plasma obtained from healthy subjects as previously described (Zechner et al., 1984). All subjects were $\mathrm{L} p(\mathrm{a})$-negative in our immunoassay, and LDL fractions were additionally subjected to immunoabsorption chromatography on an anti-apo(a)-Sepharose column to remove any contaminating Lp(a). All LDL fractions were tested in Western blot analysis for the complete absence of $L p(a)$. To avoid LDL oxidation, all steps of LDL preparation were performed 
under nitrogen and in the presence of $0.5 \mathrm{mg} / \mathrm{mL}$ EDTA. In dose-response experiments, increasing concentrations of LDL $(1,5$, and $10 \mu \mathrm{g} / \mathrm{mL}$ LDL protein) were added to $0.4 \mathrm{~mL}$ of $2 \times$ concentrated medium containing $r-a p o(a)$ at a concentration of approximately $0.5-1.0 \mathrm{nM}$. Incubations were performed for $24 \mathrm{~h}$ at $37^{\circ} \mathrm{C}$ in the presence of aprotinin, leupeptin, and PMSF as protease inhibitors. In time course experiments, $0.4 \mathrm{~mL}$ of medium was incubated under conditions identical to those mentioned above with $5 \mu \mathrm{g} / \mathrm{mL}$ LDL protein. Aliquots from the association incubation were removed at various times and analyzed for $\mathrm{r}$-apo(a)/LDL complex formation by Western blotting. To study the influence of the presence of cells on the association process, dose-response experiments were also performed directly in the tissue culture plates. LDL protein $(1,5$, and $10 \mu \mathrm{g} / \mathrm{mL})$ was added in two equivalent doses, 2 and $16 \mathrm{~h}$ after transfection, to $3 \mathrm{~mL}$ of $\mathrm{r}$-apo(a)-containing medium. After an additional $8 \mathrm{~h}$ of incubation, the media were concentrated to half the volume by a Speed-Vac (Savant). r-Apo(a)/LDL complexes were visualized by immunoblotting after SDS-agarose gel electrophoresis or SDS-PAGE. In some experiments the association mixture contained potential inhibitors of complex formation. These included iodoacetamide, SDS, $n$-octyl glucoside, $\epsilon$-aminocaproic acid, and $\mathrm{NaCl}$. Depending on the protein concentration, iodoacetamide was added at a concentration of $50-250 \mathrm{mg} / \mathrm{mL}$ directly to LDL or r-apo(a)containing medium. After an overnight incubation, free iodoacetamide was removed by gel filtration and the alkylated fractions were used in association experiments as described above. The other "inhibitors" were directly added at two different concentrations to the association mixture or $4 \mathrm{~h}$ after the association experiment was started.

Immunoquantitation of r-Apo(a) in r-Apo(a)/LDL Complexes. A double-antibody DELFIA was developed for the quantitation of r-apo(a) specifically bound to LDL after association experiments. DELFIA assays were performed according to the fluorescence immunoassay from Pharmacia (Uppsala, Sweden). Multiwell DELFIA plates were coated with a polyclonal affinity-purified antibody directed against human plasma LDL that did not cross-react with Lp(a) or apo(a). After nonspecific binding sites were blocked with $250 \mu \mathrm{L}$ of $0.5 \%$ bovine serum albumin, $200-\mu \mathrm{L}$ aliquots of sample volume from the association mixtures were added to the wells. Samples were incubated for $2 \mathrm{~h}$ at ambient temperature and washed several times with DELFIA buffer $(50 \mathrm{mM}$ Tris- $\mathrm{HCl}, \mathrm{pH} 7.7)$. For detection, an Eu-labeled polyclonal antibody against human $\mathrm{Lp}(\mathrm{a})(\mathrm{K} 202)$ was added, and the plates were again incubated for $2 \mathrm{~h}$ at ambient temperature. Excess antibody was removed by washing the plates with DELFIA buffer and $200 \mu \mathrm{L}$ of enhancement solution were added according to the protocol supplied by the manufacturer. Fluorescence was determined after $15 \mathrm{~min}$ in a DELFIA reader. An Lp(a) standard (Immuno AG, Vienna, Austria) was included in all experiments, and the assay was found to be linear between 1 and $100 \mathrm{ng}$ of $\mathrm{Lp}(\mathrm{a}) /$ well. To determine the total apo(a) concentration in the association mixtures, DELFIA assays were performed under the identical conditions except that the anti-Lp(a) antibody was also used for coating the plates.

Density Gradient Ultracentrifugation of $r$-Apo(a)-LDL Complexes. For separation of LDL-associated r-apo(a) from free r-apo(a), ultracentrifugation on a salt density gradient was performed according to Chung et al. (1980) with slight modifications. Cell culture medium $(4 \mathrm{~mL})$ was brought to a density of $1.21 \mathrm{~g} / \mathrm{mL}$ by addition of solid $\mathrm{KBr}$ and layered under $9 \mathrm{~mL}$ of an $0.15 \mathrm{M} \mathrm{NaCl}$ solution in Quickseal centrifugation tubes (Beckman, Palo Alto, CA). Centrifugation was carried out in a vertical rotor (Beckman VTi 65) at $4^{\circ} \mathrm{C}$ at $65000 \mathrm{rpm}$ for $90 \mathrm{~min}$. The densities of the fractions were estimated by measuring the refractive index. After ultracentrifugation, gradient fractions were dialyzed and analyzed for apo(a) and apoB.

\section{RESULTS}

Transferrinfection of Apo(a) cDNA Constructions. Figure 1 exhibits the apo(a) cDNA constructions used for transferrinfection in cos-7 cells. All clones contained the 5'-UTR, the coding region for the signal peptide, the kringle $V$ domain, and the protease domain, as well as the $3^{\prime}$-UTR. Additionally, the different clones contained 3 (pSG5-S), 9 (pSG5-M), 15 (pSG5-L), and 18 (pSG5-XL) kringle IV repeats. In some experiments two additional constructions were used which are designated pSG5-3.0 and pSG5-3.6. Kringle IV-36 with the unique unpaired Cys-4057 and kringle IV-37 were present in all constructions. All apo(a) cDNA sequences were cloned into pSG5, a eukaryotic expression vector under the control of the SV 40 promoter. After transferrinfection of these clones into preconfluent cos-7 cells, cell lysates and cell media were analyzed for the presence of $r$-apo(a) by SDS-agarose gel electrophoresis or SDS-PAGE and immunochemical detection using polyclonal antisera specific for apo(a). Peptides resulting from transfection of cells with plasmids pSG5-S, pSG5-M, pSG5-L, and pSG5-XL are designated r-apo(a)-S, r-apo(a)$M, r$-apo(a)-L, and r-apo(a)-XL, respectively. The two additional peptides derived from pSG5-3.0 and pSG5-3.6 are designated r-apo(a)-3.0 and r-apo(a)-3.6.

Generally, high concentrations of adenovirus in the transferrinfection mixture increased the amount of $r$-apo(a) synthesized and secreted by the cells. However, high virus titers also caused cell death. Therefore, the influence of the virus concentration in the transferrinfection procedure was optimized with regard to cell viability and transfection efficiency. Control transfections with $\beta$-galactosidase expression plasmids and subsequent enzyme assays revealed that, under the conditions used in these studies, between $30 \%$ and $50 \%$ of the cells received plasmid DNA. At these transfection efficiencies, $r$-apo(a) synthesis and secretion was found to range from 4 to $10 \mathrm{pg}$ cell $^{-1}(24 \mathrm{~h})^{-1}$ over a time period of 48 $\mathrm{h}$, as quantitated by apo(a) ELISA. Time course experiments of $\mathrm{r}$-apo(a)-L secretion after transferrinfection of $\mathrm{pSG} 5-\mathrm{L}$ into cos-7 cells revealed the highest expression of $\mathrm{r}$-apo(a)- $\mathrm{L}$ 2 days after transferrinfection after normalization to the total cellular DNA concentrations per dish. After that time point, synthesis and secretion declined.

Figure 2 displays an immunoblot after SDS-PAGE under nonreducing conditions of $\mathrm{r}$-apo(a) from lysates and media of transfected cos- 7 cells. The signal obtained from $r$-apo(a)-S was weak and only detectable in the cell medium when the polyclonal antiserum K271 was used for immunodetection (Figure 2A). Strong bands, however, were obtained for the r-apo(a)-M-, r-apo(a)-L-, and r-apo(a)-XL-expressing constructions (Figure 2B). Two intracellular $r$-apo(a) signals of different molecular weight were present. Only a single band was detected in the cell medium, which corresponded to the high molecular weight intracellular form. To test if the smaller intracellular $\mathrm{r}$-apo(a) signal represented an $\mathrm{r}$-apo(a) precursor, pSG5-L transfected cells were treated with monensin. This antibiotic inhibits protein transport within the Golgi apparatus, leaving $\mathrm{r}$-apo(a) only glycosylated to the extent that glycosylation occurred in the endoplasmic reticulum. When analyzed by SDS-PAGE and immunoblotting, intracellular $r$-apo(a)-L from monensin-treated cells exhibited the same 

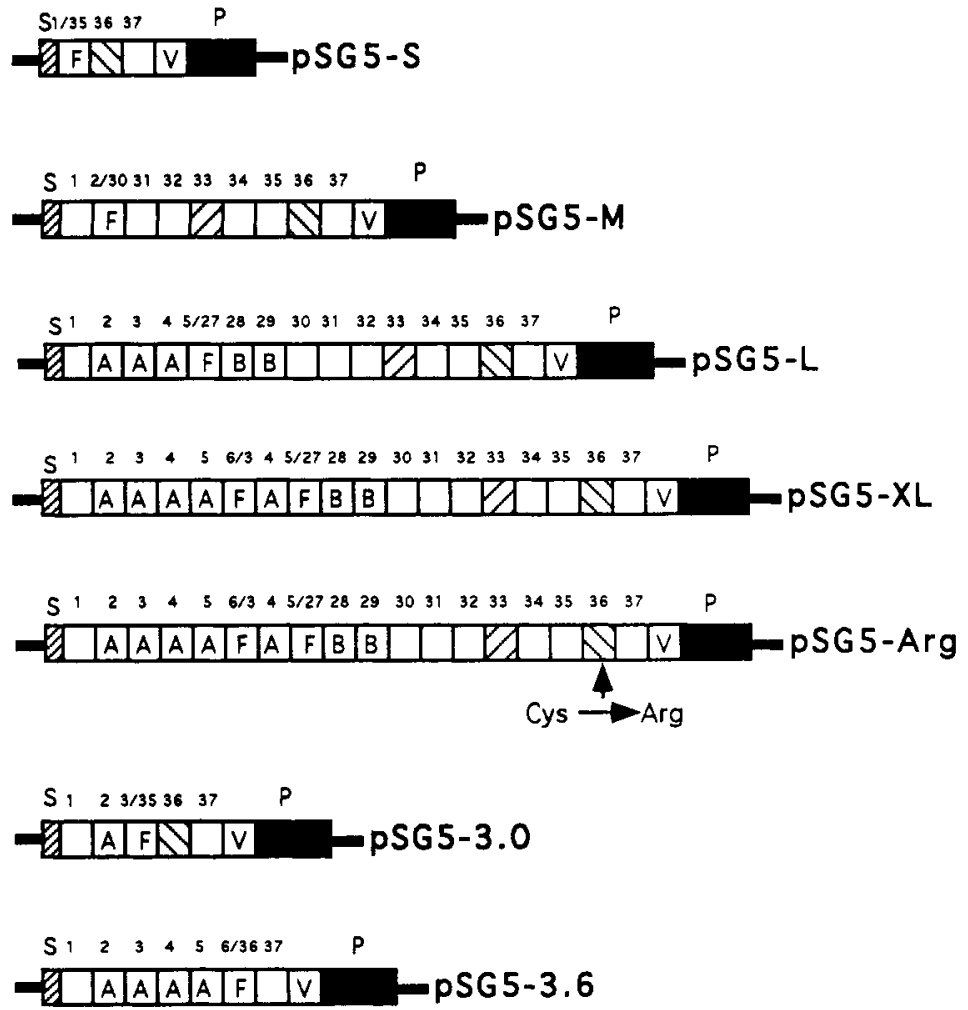

FIGURE 1: Apo(a) cDNA constructions used for transferrinfection. All constructions are compared to the apo(a)-cDNA structure published by McLean et al. (1987). All clones include the $5^{\prime}$ UTR, the coding region for the signal peptide (S), the kringle V domain (V), the protease domain (P), the $3^{\prime}$ UTR, and variable numbers of kringle IV repeats. The kringle IV repeats in each construction are numbered and labeled according to their structural characteristics as A repeats, B repeats, or unique sequence repeats as suggested by McLean et al. (1987). Fused kringle IV repeats are also indicated (F). pSG5-Arg had the identical structure as pSG5-XL except that a single free cysteine residue in kringle IV-36 (Cys-4057) was replaced by arginine using site-directed mutagenesis.

molecular weight as the smaller, intracellular isoform from untreated cells (Figure $2 \mathrm{C}$ ). This suggested that the smaller protein within transfected cos-7 cells represented a partially glycosylated or unglycosylated precursor protein.

The molecular sizes of intra- and extracellular protein bands increased with the number of kringle IV sequences within apo(a) cDNA constructions. The smallest clone yielded a mature protein with an apparent molecular mass of approximately $300 \mathrm{kDa}$, whereas the largest construction with 18 kringle IV sequences yielded an apparent molecular mass of $550 \mathrm{kDa}$. No obvious differences were observed with regard to signal intensity among $r$-apo(a)-M, r-apo(a)-L, and $\mathrm{r}$ apo(a)-XL. However, r-apo(a)-S, with only three "unique sequence" kringle IV repeats, was only poorly detected by Western blotting using the polyclonal antiserum K271. To investigate if the poor detection of $r$-apo(a)-S was due to poor reactivity of $K 271$ or a decreased expression efficiency of this expression plasmid, a recently developed antibody specific for kringle V of apo(a) (Huby et al., 1994) and a newly developed polyclonal antiserum (K202) were used in Western blotting experiments. With these reagents identical expression efficiency was observed for r-apo(a)-S compared to the other clones. This suggested that the polyclonal antiserum K271 was mainly directed against kringle IV sequences not present on r-apo(a)-S. When the monoclonal antibody $1 \mathrm{~A} 2$ was used for immunoblotting or ELISA analysis, r-apo(a)-S was not detected at all. The binding site of this monoclonal antibody was recently mapped to kringle IV sequences of apo(a) which are not present on r-apo(a)-S (H. Dieplinger et al., submitted for publication).
Synthesis and Secretion of $r$-Apo(a) by Glycosylation Mutants. To study the influence of N-glycosylation on the synthesis and secretion of $r$-apo(a), glycosylation-defective Chinese hamster ovary (CHO) cells ( Lec 1, Lec 2, and Lec 8 ) and the wild-type parental cell line of these mutant lines (Pro5-) were transfected with pSG5-L. r-Apo(a)-L synthesis and secretion were then analyzed by Western blotting and ELISA quantitation. In preliminary experiments it was established that the lack of carbohydrates or sialic acid does not change the immunoreactivity of the polyclonal antiserum or the monoclonal antibody $1 \mathrm{~A} 2$ used in these studies $(\mathrm{H} . \mathrm{J}$. Menzel and G. M. Kostner, personal communication). Lec 1 cells lack glycosyltransferase $\mathrm{T} 1$ so that $\mathrm{N}$-linked carbohydrate addition is blocked at the $\mathrm{Man}_{5} \mathrm{GlcNAc} \mathrm{Assn}_{2}$ intermediate (Stanley et al., 1975). Lec 2 cells exhibit a drastic reduction in the transport of CMP-sialic acid into the Golgi compartment, resulting in severely decreased sialylation of proteins (Deutscher et al., 1984). Lec 8 cells exhibit a drastic reduction in the ability to transport UDP-galactose into the Golgi compartment (Deutscher \& Hirschberg, 1986). To provide these experiments with an internal control for transfection efficiencies, pSG5-L was cotransfected with a human growth hormone expression plasmid, pGH. Human growth hormone is not glycosylated, and therefore, its expression is not influenced by the glycosylation defects in the CHO cell lines used. Table 1 summarizes the results. Compared to the wild-type $\mathrm{CHO}$ cell line, Pro 5-, Lec 1 cells secreted $55.6 \% \pm 9.4 \%$ as much $\mathrm{r}$-apo(a)- $\mathrm{L}$ when the expression was normalized to growth hormone expression. This indicated that addition of NAcGlc was important for proper apo(a) 
A

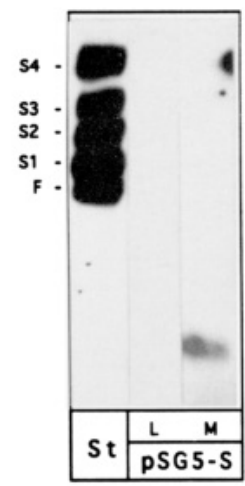

B

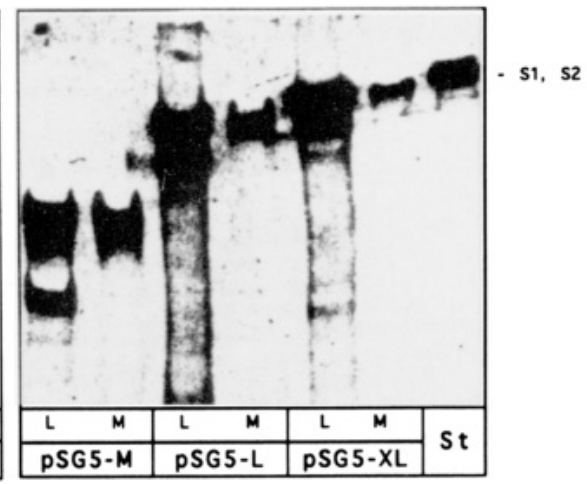

C

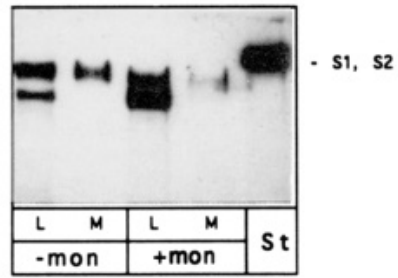

FIGURE 2: Analysis of $r$-apo(a) in the media and cell lysates of transfected cos-7 cells. Cos-7 cells were transfected with pSG5-S, pSG5-M, pSG-L, or pSG5-XL. Twenty-four hours after transfection, cell media were collected and cells were harvested and lysed. (A) Media (M) and lysates (L) of cells transfected with pSG-S were subjected toSDS-1.5\% agarose gel electrophoresis under nonreducing conditions and transferred to nitrocellulose filters. Immunodetection was performed using a rabbit anti-human apo(a) antiserum (K271) and an enhanced chemiluminescent detection system. To estimate the molecular sizes of the bands, an apo(a) size standard was included (St) and is labeled on the left. (B) Media (M) and lysates (L) of cells transfected with pSG5-M, pSG5-L, and pSG-XL were subjected to denaturing SDS-6.6\% PAGE and transferred to nitrocellulose filters. Immunodetection was performed using a monoclonal apo(a) antibody (clone 1A2) and an enhanced chemiluminescent detection system. To estimate the molecular sizes of the bands, an apo(a) size standard was included (St) and is labeled on the right. (C) Media (M) and lysates (L) of cells transfected with pSG5-L and cultured in the presence $(+$ mon) or absence $(-$ mon) of $1 \mu \mathrm{M}$ monensin were subjected to $6.6 \%$ denaturing SDS-6.6\% PAGE and transferred to nitrocellulose filters. Immunodetection was performed using a monoclonal apo(a) antibody (clone 1A2) and an enhanced chemiluminescent detection system. To estimate the molecular sizes of the bands, an apo(a) size standard was included (St) and is labeled on the right.

Table 1: Secretion of r-Apo(a)-L into the Medium of Transfected Glycosylation Defective CHO Cell Lines ${ }^{a}$

\begin{tabular}{lcccc}
\hline \multicolumn{1}{c}{ cell line } & $\begin{array}{c}\text { cell } \\
\text { number }\end{array}$ & $\begin{array}{c}\mathrm{hGH}^{c} \\
(\mathrm{cpm})\end{array}$ & $\begin{array}{c}\mathrm{apo}(\mathrm{a})^{d} \\
(\mathrm{ng} / \mathrm{mL})\end{array}$ & $\begin{array}{c}\text { apo(a) } \\
(\% \pm \mathrm{SD})\end{array}$ \\
\hline Pro 5- (wild type) & 370000 & 11282 & 733 & 100 \\
Lec 1 & 464000 & 10172 & 438 & $55.6 \pm 9.4$ \\
Lec 2 & 460000 & 10379 & 1050 & $151.9 \pm 66.4$ \\
Lec 8 & 459000 & 10708 & 583 & $89.2 \pm 25.8$ \\
\hline
\end{tabular}

${ }^{a}$ Wild-type and glycosylation-defective $\mathrm{CHO}$ cells were transfected with pSG5-L and the secretion of apo(a) into the medium after incubation for $24 \mathrm{~h}$ was measured by ELISA. Results of one representative experiment are shown. The amount of apo(a) secreted from mutant cells in relation to Pro5- (percent) was calculated from four separate experiments. ${ }^{b}$ Lec 1 , lack of glycosyltransferase T1; Lec 2, decreased sialic acid addition; Lec 8 , decreased galactose addition. ${ }^{c}$ Human growth hormone expression served as internal control for transfection efficiencies. ${ }^{d}$ Apo(a) concentrations are normalized to $\mathrm{hGH}$ activities.

secretion. Unexpectedly, transfected Lec 2 cells secreted $151.9 \% \pm 66.4 \%$ as much of $\mathrm{r}$-apo(a)- $\mathrm{L}$ compared to the expression in Pro $5^{-}$cells, suggesting that addition of sialic acid as a last step in carbohydrate modification was not necessary for the efficient secretion of $r$-apo(a). Lec 8 cells
A

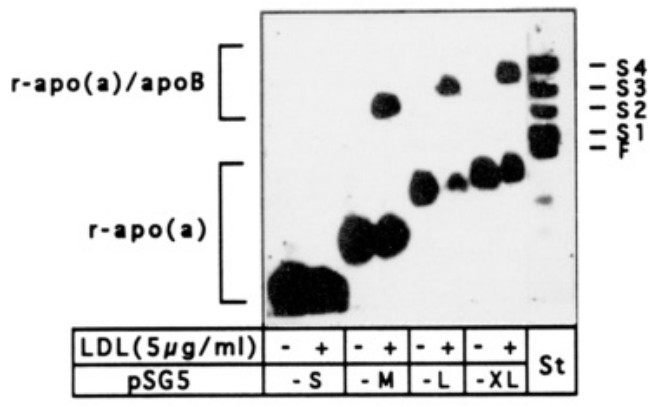

B

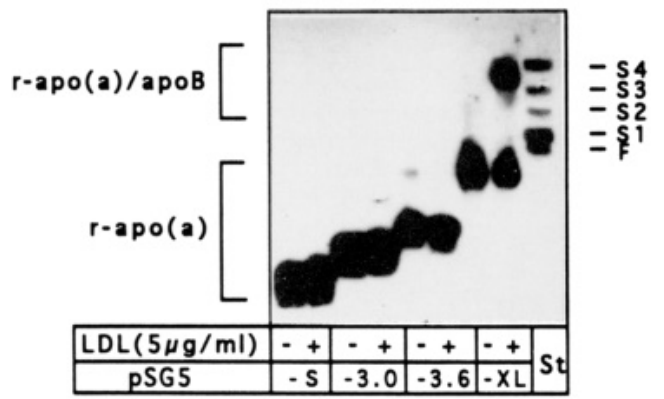

FIGURE 3: Extracellular association of $r$-apo(a) of variable length with human plasma LDL. (A) Cos-7 cells were transfected with pSG5-S, pSG5-M, pSG5-L, and pSG5-XL. (B) Cos-7 cells were transfected with pSG5-S, pSG5-3.0, pSG5-3.6, and pSG5-XL. In both experiments $5 \mu \mathrm{g} / \mathrm{mL}$ LDL (protein) was added in two equal aliquots 2 and $16 \mathrm{~h}$ after transfection directly to the culture dishes. Twenty-four hours after transfection, the cell medium was collected and concentrated two times in the presence of protease inhibitors. Aliquots of each association mixture were subjected to SDS- $1.5 \%$ agarose gel electrophoresis under nonreducing conditions. After protein transfer to nitrocellulose filters, apo(a)-specific bands were visualized using a rabbit anti-human apo(a) antiserum (K202) and an enhanced chemiluminescent detection system. The positions of free apo(a) and apo(a)-apoB complexes are indicated on the left. To estimate the molecular sizes of the bands, an apo(a) size standard was included (St) and is labeled on the right.

expressed and secreted similar levels of $\mathrm{r}-\mathrm{apo}(\mathrm{a})$ - $\mathrm{L}$ as wildtype Pro $5^{-}$cells.

$r$-Apo(a) Binding to $L D L$. An important objective of this study was to investigate the ability of r-apo(a) to bind to LDL. Therefore, extracellular association of r-apo(a) of variable sizes and carbohydrate contents to human plasma LDL was analyzed in an in vitro incubation system $24 \mathrm{~h}$ after transferrinfection. Before association experiments were conducted, the concentration of r-apo(a) was quantitated by ELISA. Freshly prepared human LDL at different concentrations was added directly to the culture plates or to the medium after it was removed from the cells. Association of $\mathrm{r}$-apo(a) with LDL was analyzed at different time points by agarose gel electrophoresis and immunoquantitation, as well as density gradient ultracentrifugation. Figure 3A displays an immunoblot after SDS-agarose gel electrophoresis that indicates the complex formation of r-apo(a) of variable sizes with LDL under nonreducing conditions. r-Apo(a)/LDL complexes were identified by their retarded electrophoretic mobility compared to free r-apo(a). Complexes could be identified with specific antibodies to apo(a) or apoB. Approximately the same amounts of complex formation was observed when r-apo(a)-M, r-apo(a)-L, or r-apo(a)-XL was incubated with human plasma LDL. In contrast, $r$-apo(a)-S containing only three kringle IV repeats did not associate with LDL at all. 


\begin{tabular}{|c|c|c|c|}
\hline $\begin{array}{c}\text { time of } \\
\text { incubation }(h)\end{array}$ & $\begin{array}{c}\text { r-apo(a)-L } \\
{[\mathrm{ng} / \mathrm{mL}]}\end{array}$ & $\begin{array}{c}\text { apo-B in } \\
\text { complex }[\mathrm{ng} / \mathrm{mL}]\end{array}$ & $\begin{array}{c}\text { complex } \\
\text { formation (\%) }\end{array}$ \\
\hline 0.25 & 390.8 & 58.3 & 15 \\
\hline 0.5 & 407.4 & 71.7 & 18 \\
\hline 2 & 396.9 & 99.4 & 25 \\
\hline 4 & 388.8 & 127.1 & 33 \\
\hline 6 & 382.0 & 150.9 & 39 \\
\hline 8 & 383.6 & 166.0 & 43 \\
\hline 16 & 384.5 & 218.7 & 57 \\
\hline 24 & 386.5 & 237.3 & 61 \\
\hline
\end{tabular}

${ }^{a} \mathrm{r}-\mathrm{Apo}(\mathrm{a})-\mathrm{L}$ and apoB were quantitated by double antibody DELFIA analyses.

To study the requirements of certain kringle structures for the complex formation with LDL in more detail, additional constructs were created and transfected into cos-7 cells, and the expressed $\mathrm{r}$-apo(a) variants were used in association experiments. Figure 3B demonstrates that $\mathrm{r}$-apo(a)-3.0, which contains five kringle IV "type A" structures including kringle IV-36 and kringle IV-37 but lacks the "unique kringle" sequences (kringle IV-31 through 35 ), does not form complexes with LDL. A construct resulting in a r-apo(a) with seven kringle IV sequences including kringle IV-36 and 37 [ $r$-apo(a)3.6] but lacking the "unique kringle" structures (kringle IV31 through 36) is also unable to associate with the human plasma LDL.

The amount of r-apo(a)-L complexed to LDL was also determined directly from association mixtures by doubleantibody DELFIA analysis. Medium obtained from cos-7 cells transfected with pSG5-L, containing $0.4-0.8 \mu \mathrm{g} / \mathrm{mL}$ r-apo(a), was mixed with freshly prepared human plasma $\mathrm{LDL}$ and incubated for $24 \mathrm{~h}$ at $37^{\circ} \mathrm{C}$. The relative amount of $r$-apo(a) within r-apo(a)/LDL complexes was quantitated by DELFIA assays performed in duplicate. In DELFIA-1, anti-Lp(a) was used as coating antibody and detection antibody. In DELFIA-2, anti-LDL was used as coating antibody and anti-Lp(a) was used as detection antibody (described in Experimental Procedures). Therefore, DELFIA-1 quantitated the total amount of r-apo(a)-L present in the incubation mixture, whereas DELFIA-2 detected only $\mathrm{r}$-apo(a)-L within r-apo(a)/LDL complexes. By this method, $30-75 \%$ of r-apo(a)-L was detected within the complex after 24-h incubations, depending on the amount of apo(a) and LDL protein present in the mixture.

Table 2 demonstrates the kinetics of the association between $\mathrm{r}$-apo(a)-L and LDL. Complex formation was quantitated by double-antibody DELFIA analysis. Complex formation could be detected after $0.5 \mathrm{~h}$ of incubation and increased up to $24 \mathrm{~h}$. In this experiment incubations were performed in the absence of cells, indicating that the presence of cells is not a prerequisite for complex formation. In a dose-response experiment, increasing amounts of freshly prepared human LDL were added directly to r-apo(a)-L-containing medium in the cell culture dishes. As demonstrated in Figure 4 more than $50 \%$ of r-apo(a)-L was complexed to LDL when the LDL was present in a 2 -fold molar excess $(1 \mu \mathrm{g} / \mathrm{mL}$ of LDL protein), indicating the high specificity of the process. The presence of $5 \% \beta$-mercaptoethanol in the incubation mixture completely inhibited complex formation. In the absence of $\mathrm{r}$-apo(a)-L no signals were detected, whereas in the absence of LDL in the incubation mixture only uncomplexed r-apo(a)-L was found when anti-apo(a) antiserum was used for immunodetection (not shown).

To demonstrate that r-apo(a)-L was in fact lipoproteinassociated in the r-apo(a)/LDL complex, an association mixture containing $5 \mu \mathrm{g} / \mathrm{mL}$ LDL protein, corresponding to

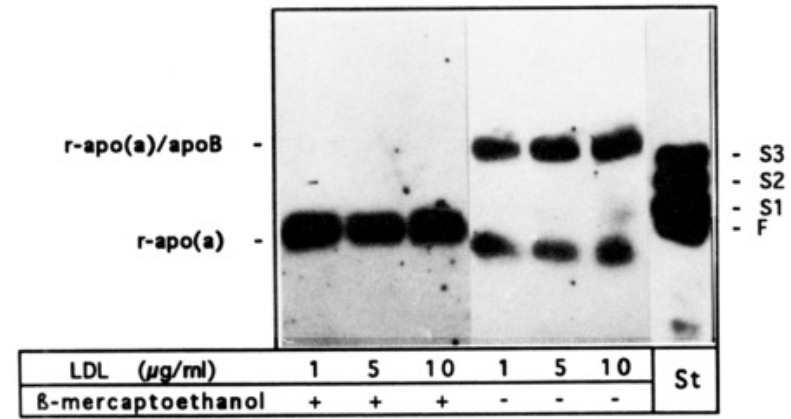

FIGURE 4: Extracellular association of $\mathrm{r}$-apo(a)-L with human plasma LDL. Cos-7 cells were transfected with pSG5-L. The indicated amounts of LDL (protein) were added directly to the cell culture dishes in two equal aliquots 2 and $16 \mathrm{~h}$ after transfection. Twentyfour hours after transfection, the cell medium was collected and concentrated two times in the presence of protease inhibitors. Aliquots of each association mixture were subjected to SDS-1.5\% agarose gel electrophoresis under nonreducing conditions or in the presence of $5 \% \beta$-mercaptoethanol. After protein transfer to nitrocellulose filters, apo(a)-specific bands were visualized using a rabbit anti-human apo(a) antiserum (K271) and an enhanced chemiluminescent detection system. The positions of free apo(a) and apo(a)-apoB complexes are indicated on the left. To estimate the molecular sizes of the bands, an apo(a) size standard was included (St) and is labeled on the right.

a 5:1 molar excess of LDL over r-apo(a)-L, was analyzed by density gradient ultracentrifugation. Following fractionation of the gradient, $\mathrm{r}$-apo(a) and apoB were quantitated in all samples by ELISA. Figure 5 demonstrates that r-apo(a)-L can be detected at a buoyant density between $d=1.08$ and $1.13 \mathrm{~g} / \mathrm{mL}$, which is typical for $\mathrm{Lp}(\mathrm{a})$. Between $20 \%$ and $60 \%$ of $\mathrm{r}$-apo(a)- $\mathrm{L}$ was found in the $\mathrm{Lp}$ (a) density region, and the rest was detected as free $r$-apo(a)-L in the bottom fraction. These results were in good agreement with data obtained from DELFIA analyses. ApoB was found in a density range between buoyant densities $d=1.02$ and $1.13 \mathrm{~g} / \mathrm{mL}$, covering the density region of $L D L$ and $L p(a)$. In the absence of $L D L$, all r-apo(a)-L was recovered in the lipid-free bottom fraction.

Role of the Carbohydrate Moiety of r-Apo(a) for Its Association with LDL. The contribution of the carbohydrate content of r-apo(a) to the complex formation with LDL was studied using glycosylation-defective Lec 1 , Lec 2, and Lec 8 cells transfected with pSG5-L. By SDS-agarose gel electrophoresis and immunoblotting, mixtures of free and complexed r-apo(a)-L were detected, which were generated in a 24-h incubation period (Figure 6). The apparent sizes of uncomplexed r-apo(a)-L differ slightly due to the differences in their carbohydrate content. All of the different glycosylation forms of r-apo(a)-L tested formed complexes with LDL. Table 3 summarizes the results of the double-antibody DELFIA analyses. The relative percentage of $\mathrm{r}$-apo(a)- $\mathrm{L}$ associated with LDL increased with increasing LDL concentrations from $40 \%$ to $60 \%$. No significant difference in the amounts of r-apo(a)-L within the complexes was found for all three glycosylation variants when compared to wild-type r-apo(a)L. This indicated that complete glycosylation of r-apo(a) was not a prerequisite for complex formation.

Role of Unpaired Cys Residues on r-Apo(a) or LDL for Association. The functional importance of free cysteine residues on r-apo(a) for complex formation was studied by chemical modification of free cysteine residues by iodoacetamide on LDL and r-apo(a), respectively. Following alkylation, excess iodoacetamide was removed by gel filtration, and normal or modified $\mathrm{r}$-apo(a)-XL containing medium was mixed with normal or modified LDL. Figure 7 summarizes the results of the double-antibody DELFIA analysis. Alkylation of Cys residues on LDL decreased r-apo(a)-LDL 


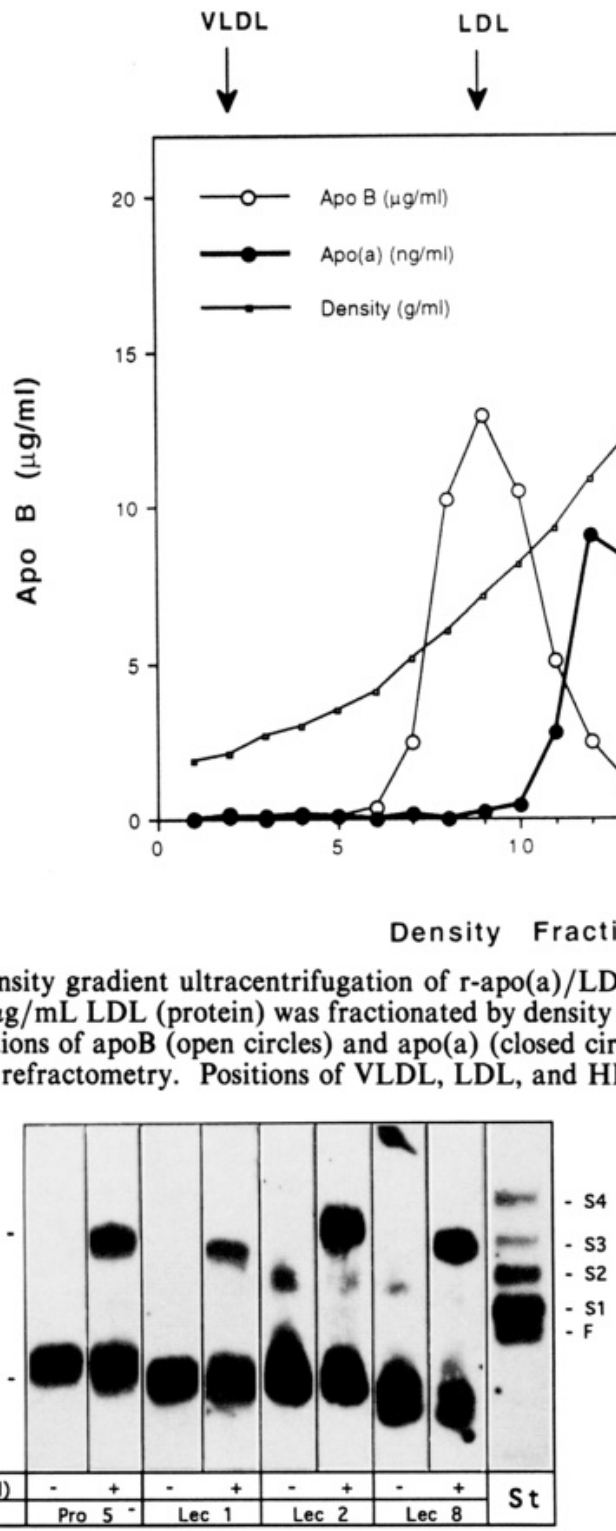

FIGURE 6: Influence of the carbohydrate moiety of r-apo(a) on complex formation with LDL. Wild-type $\mathrm{CHO}$ cells (Pro5-) and CHO cells exhibiting different defects in N-glycosylation (Lec 1, Lec 2, and Lec 8 cells; see Experimental Procedures) were transfected with pSG5-L. LDL $(5 \mu \mathrm{g} / \mathrm{mL}$ protein, $+\mathrm{LDL})$ was added directly to the cell culture dishes in two equal aliquots 2 and $16 \mathrm{~h}$ after transfection. Control plates with no LDL added (-LDL) were otherwise treated identically as +LDL plates. Twenty-four hours after transfection, the cell media were collected and aliquots were subjected to nondenaturing SDS-1.5\% agarose gel electrophoresis. After protein transfer to nitrocellulose filters, apo(a)-specific bands were visualized using a rabbit anti-human apo(a) antiserum (K271) and an enhanced chemiluminescent detection system. The positions of free apo(a) and apo(a)-apoB complexes are indicated on the left. To estimate the molecular sizes of the bands, an apo(a) size standard was included (St) and is labeled on the right.

association by $50 \%$. If free cysteine residues were modified in LDL and r-apo(a)-XL, complex formation was decreased by more than $90 \%$.

To investigate if the extracellular association between $\mathrm{r}$-apo(a) and LDL was dependent on the presence of a single free cysteine residue in $\mathrm{r}$-apo(a) at a position corresponding to amino acid residue 4057 of the mature protein (McLean et al., 1987) this position was altered to arginine by in vitro mutagenesis of pSG5-XL. The presence of the correct singlebase mutation was verified by DNA sequencing, and the mutated clone (pSG5-Arg) was transfected into cos- 7 cells by transferrinfection. The expression of r-apo(a)-Arg and its
HDL 


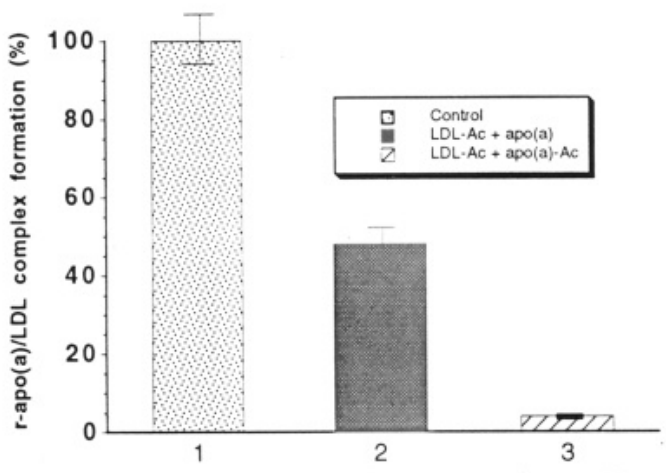

FIGURE 7: Extracellular complex formation of r-apo(a)-XL with LDL after alkylation of free cysteine residues. Cos-7 cells were transfected with pSG5-XL and the resulting cell medium was used in association experiments after treatment with iodoacetamide (for details, see Experimental Procedures). Similarly, LDL was also modified by iodoacetamide treatment. (1) In a control experiment, unmodified medium and unmodified LDL were used in the association experiment. (2) Modified LDL was incubated with unmodified medium. (3) Modified LDL was incubated with modified medium. Incubation conditions were identical as described above and complex formation was monitored by double-antibody DELFIA analyses. Complex formation in the control mixture was set to $100 \%$.

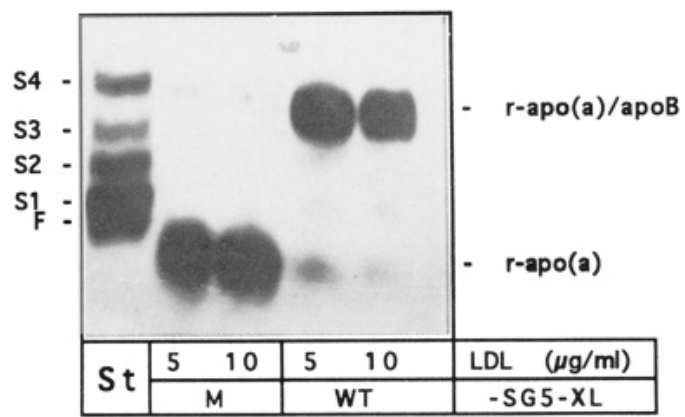

FIGURE 8: Extracellular association of r-apo(a)-Arg (Cys4057 $\rightarrow$ Arg) with LDL. Cos-7 cells were transfected with wild-type pSG5XL (WT) or mutated pSG5-Arg (M). In the mutated clone Cys4057 was replaced by Arg using site-directed mutagenesis. LDL ( 5 or $10 \mu \mathrm{g}$ of protein $/ \mathrm{mL}$ ) was added in two equal aliquots 2 and $16 \mathrm{~h}$ after transfection. Twenty-four hours after transfection the cell media were collected and aliquots were subjected to nondenaturing SDS-agarose gel electrophoresis. After protein transfer to nitrocellulose filters, apo(a)-specific bands were visualized using a rabbit anti-human apo(a) antiserum (K271) and an enhanced chemiluminescent detection system. The positions of free apo(a) and apo(a)apoB complexes are indicated on the right. To estimate the molecular sizes of the bands, an apo(a) size standard was included (St) and is labeled on the left.

LDL, although no unpaired Cys residue was present in this r-apo(a) species.

"Noncovalent" Interaction between $r-A p o(a)-L$ and $L D L$. To study the nature of interaction between $\mathrm{r}$-apo(a) and LDL independent of the formation of a disulfide bond in more detail, complex formation was measured by double-antibody DELFIA in the presence of high salt concentrations, detergents, and $\epsilon$-aminocaproic acid, using wild-type r-apo(a)-XL or its mutated homologue $\mathrm{r}$-apo(a)-Arg. The results are displayed in Table 4. $\epsilon$-Aminocaproic acid can completely prevent complex formation of r-apo(a)-XL or r-apo(a)-Arg with LDL when added at a concentration of $50 \mathrm{mM}$. However, when the inhibitor was added $4 \mathrm{~h}$ after the association incubation was initiated, already-formed complexes between r-apo(a)$\mathrm{XL}$ and LDL were only partly dissociated. In contrast, complexes between r-apo(a)-Arg and LDL were sensitive to $\epsilon$-aminocaproic acid even after the 4-h preincubation period. This suggesting that r-apo(a)-XL bound to LDL by disulfide bridges was not sensitive to the treatment with $\epsilon$-aminocaproic acid, whereas the noncovalent interaction between $\mathrm{r}$-apo(a)Arg and LDL was easily dissociated. High salt concentration did not affect complex formation of wild-type or mutant r-apo(a) at a sodium chloride concentration of up to $10 \%$. In contrast, detergents, both ionic and nonionic, strongly decreased the efficiency of complex formation for both r-apo(a)$\mathrm{XL} / \mathrm{LDL}$ complexes and r-apo(a)-Arg/LDL complexes.

\section{DISCUSSION}

$\mathrm{Lp}(\mathrm{a})$ is a very atherogenic lipoprotein. The concentration of $\mathrm{Lp}(\mathrm{a})$ is essentially unaffected by dietary or drug treatment (Kostner, 1988). Understanding the biosynthesis of $L p(a)$ is therefore important and may provide the rationale for the development of drug intervention protocols to control $\mathrm{Lp}(\mathrm{a})$ levels in human plasma. We and others have demonstrated that $\mathrm{Lp}(\mathrm{a})$ plasma levels are determined by the rate of synthesis and not by the catabolic rate (Krempler et al., 1983; Rader et al., 1993). Recent work by others using transgenic mice (Chiesa et al., 1992; Linton et al., 1994) and primary cultures of baboon liver cells suggested that $\mathrm{Lp}$ (a) assembly may occur in the plasma compartment (White et al., 1993). These observations were substantiated very recently by studies in transfected cells where extracellular interaction of r-apo(a) with LDL was reported (Chiesa et al., 1992; Koschinsky et al., 1993). Several mechanisms of interaction between apo(a) and LDL were suggested, including ionic interaction between apo(a) and apoB as well as the formation of a disulfide bond between the apolipoproteins involving Cys-4057 (Sommer et al., 1991; Guevara et al., 1993; McLean et al., 1987). Two recent studies demonstrated that Cys-4057 is directly involved in $\mathrm{Lp}$ (a) assembly (Koschinsky et al., 1993; Brunner et al., 1993).

In the present study we examined the following questions: First, does the number of kringle IV sequences affect the synthesis and secretion of apo(a) or its ability to associate with LDL? Second, is the presence of kringle IV-36 in r-apo(a) sufficient for $\mathrm{r}$-apo(a)/LDL association? Third, is a single free $\mathrm{SH}$ group on kringle IV-36 of r-apo(a) necessary for its association with apoB? Fourth, how are r-apo(a) synthesis, secretion, and complex formation affected by the carbohydrate moiety of apo(a)?

To address these questions, a high-level expression system for r-apo(a) was required to facilitate an accurate measurement of r-apo(a) in its free form and within r-apo(a)/LDL complexes by DELFIA quantitation and Western blotting analysis. To achieve this, apo(a) cDNAs of variable length were cloned into the eukaryotic expression vector pSG5 and transiently transfected into cos-7 cells. "Transferrinfection" (Wagner et al., 1990; Zatloukal et al., 1992; Cotten et al., 1992) was chosen for the transfection protocol. This procedure relies on receptor-mediated DNA uptake via the transferrin or the adenovirus receptor of cells. Apo(a) cDNA constructions, a polylysine-transferrin conjugate, and the replication-defective adenovirus $\mathrm{dI} 313$ form a complex particle that is taken up by receptor-mediated endocytosis. After internalization and formation of coated vesicles, the adenovirus coat proteins lyse the newly formed endosomes. Thus lysosomal degradation of the complex is bypassed, resulting in a high copy number of plasmids and highly efficient expression of $r$-apo(a) in the cells.

By this method r-apo(a) was secreted in its glycosylated form. Highest rates of r-apo(a) synthesis were observed between 24 and $48 \mathrm{~h}$ after transfection. ELISA assays using a polyclonal coating antiserum and a monoclonal detection antibody determined that r-apo(a) molecules with 9,15 , or 18 kringle IV repeats were expressed at high concentrations. The r-apo(a)-S, however, with only three kringle IV sequences, was poorly detected by the polyclonal antiserum K271. The 
Table 4: Effect of $\epsilon$-Aminocaproic Acid, $\mathrm{NaCl}, \mathrm{SDS}$, and $n$-Octyl Glucoside on the Association of $\mathrm{r}$-Apo(a)-XL and $\mathrm{r}$-Apo(a)-Arg with $\mathrm{Human}$ Plasma LDL

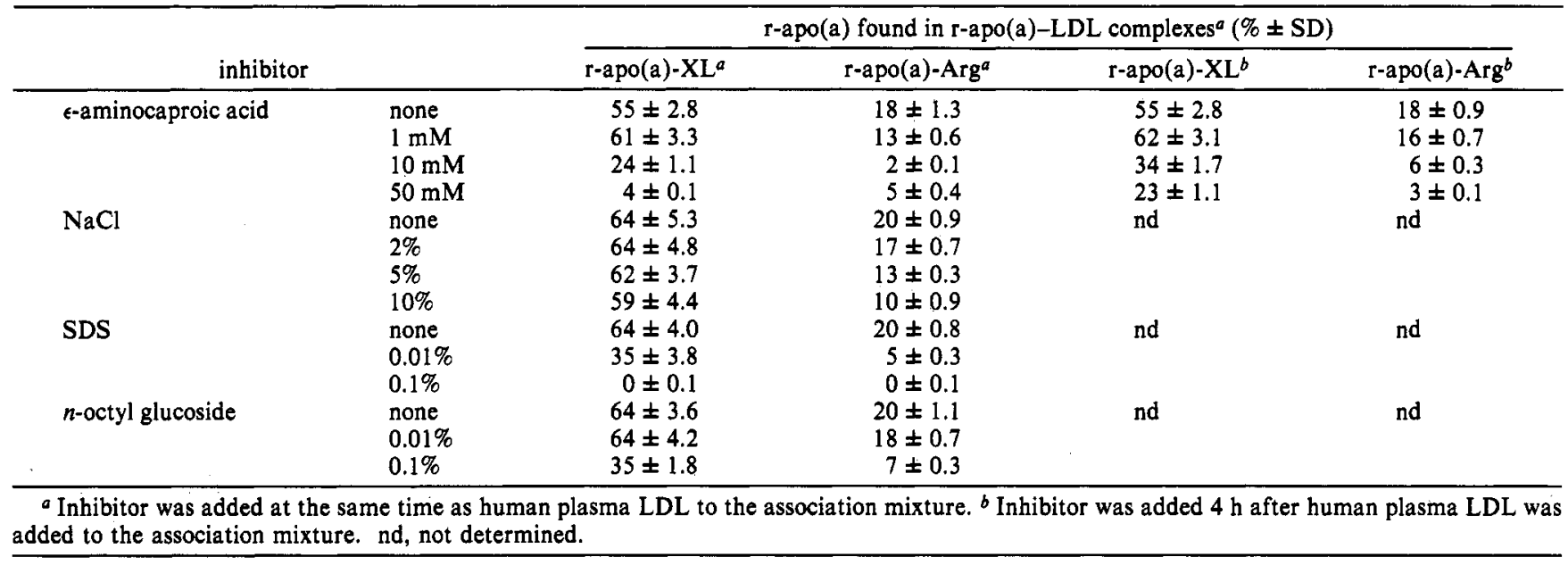

monoclonal antibody $1 \mathrm{~A} 2$ did not detect the resulting recombinant protein at all, because it is directed against an epitope in a "type A" kringle IV repeat not present on r-apo(a)-S (Dieplinger et al., submitted for publication). Experiments with a monoclonal antibody directed against kringle $V$ sequences of apo(a) (Huby et al., 1994) or a new polyclonal antiserum (K202) revealed, however, that rapo(a)-S is synthesized and secreted at normal concentrations.

Apo(a) is a highly glycosylated protein with a high sialic acid content. To study the influence of the carbohydrate content on the synthesis and secretion of apo(a), $\mathrm{CHO}$ cell lines with well-characterized glycosylation defects were transfected and r-apo(a) production was monitored. The antibodies used in this study have been shown to react identically to apo(a) species with varying carbohydrate contents (H. J. Menzel and G. M. Kostner, personal communication). The results suggested that sialic acid residues on $r$-apo(a) are not a prerequisite for proper secretion of the protein. In fact, transfected cells with a severely decreased ability to add sialic acid residues produced about $50 \%$ more $r$-apo(a) than transfected cells without such a defect. This could be caused by the bypassing of certain glycosylation steps and therefore a faster processing of $r$-apo(a) in the Golgi apparatus. In contrast to sialylation, addition of $\mathrm{N}$-acetylglucosamine by glycosyltransferase $\mathrm{T} 1$ in the Golgi compartment seems to be important for proper secretion since transfected cells with a defect in this processing step secreted only about half the amount of $r$-apo(a) as transfected wild type cells did.

r-Apo(a) secreted by transiently transfected normal cos-7 cells readily associated with $\mathrm{LDL}$ when approximately equimolar amounts were combined and the mixture was incubated at $37^{\circ} \mathrm{C}$. Complex formation was observed when LDL was directly added to the medium in the culture plates or when it was added to medium that had been removed from the cells. However, complex formation was always slightly more efficient in the presence of cells. A possible explanation for this might be that, in the presence of cells, once the r-apo(a) is secreted, it can immediately associate with LDL. This complex formation "in statu nascendi" might protect $r$-apo(a) from modification, e.g., oxidation or degradation.

The kinetics of association in our experiments differ from the data reported by Chiesa et al. (1992). Whereas these authors reported that $\mathrm{r}$-apo(a) associated with LDL in the order of minutes, it took $0.5-1 \mathrm{~h}$ to observe a significant accumulation of complexes in our experiments. This discrepancy remains unexplained but might be caused by a difference between the r-apo(a) produced in tissue culture versus apo(a) produced in transgenic mice. Another plausible explanation is that certain plasma components enhance complex formation, as has been reported by Koschinsky et al. (1993).

Complex formation was very efficient at equimolar concentrations of LDL and r-apo(a) when the complexes were analyzed in SDS-agarose gel electrophoresis and immunoblotting experiments. Association was observed for r-apo(a) molecules with 9,15 , and 18 kringle IV repeats but was undetectable in a construction with only three kringle IV repeats, despite the fact that the unique kringle containing the free cysteine residue (kringle IV-36) was present in this construction. These results indicated that the presence of the single free cysteine residue on r-apo(a) alone was not sufficient for complex formation. To substantiate these results, we analyzed additional $r$-apo(a) variants and demonstrated that the region between kringle IV-31 and kringle IV-35 is essential for complex formation. Replacement of these kringle structures by kringle IV-1 through 5 did not result in the ability to form complexes with LDL. r-Apo(a) with defects in the carbohydrate moiety exhibited similar association properties as wild-type $r$-apo(a), indicating that the negative charge contributed by the sialic acid residues does not participate in apo(a)-apoB interaction.

The apo(a)/apoB heterodimers found in native $L p(a)$ do not dissociate upon delipidation. Separation is observed only if $L p(a)$ is incubated with disulfide reducing agents such as DTT or $\beta$-mercaptoethanol. The same was true in our experiments, where complexes dissociated instantly after the addition of $5 \% \beta$-mercaptoethanol, indicating that disulfide bridge(s) stabilized the apo(a)/LDL complex. This was further substantiated by the finding that alkylation of free Cys residues on $\mathrm{LDL} / \mathrm{apoB}$ or $\mathrm{r}$-apo(a) decreased complex formation drastically, again suggesting the formation of a disulfide bridge during the association process, as has been proposed by many other studies.

To investigate the proposed role of cysteine residue 4057 on apo(a) as the site of interaction with LDL, it was replaced by arginine, utilizing site-directed mutagenesis. The resulting mutated protein, r-apo(a)-Arg, was indistinguishable from r-apo(a) with regard to its synthesis, secretion, and immunological reactivity toward all anti-apo(a) antibodies used in our laboratories. r-Apo(a)-Arg had a drastically decreased affinity to bind to LDL. Similar results were obtained in two other studies in which Cys-4057 was replaced by Ser or Gly (Koschinsky et al., 1993; Brunner et al., 1993). Small amounts 
of complexes formed also with the mutant $r$-apo(a) in our study, indicating that in addition to the formation of a disulfide bridge, noncovalent binding between $r-a p o(a)$ and LDL occurs that is independent of Cys-4057. Strong "noncovalent" interaction between apo(a) and apoB has been observed earlier (Phillips et al., 1992; Kostner \& Grillhofer, 1991). To study this "noncovalent" interaction in more detail we have performed association experiments in the presence of different potential inhibitors. Whereas $\epsilon$-aminocaproic acid and ionic as well as nonionic detergents completely abolished complex formation when present in the association experiment, high salt concentrations up to $10 \% \mathrm{NaCl}$ did not inhibit the formation of $\mathrm{r}$-apo(a)/LDL complexes. Interestingly, the "noncovalent" interaction between r-apo(a)-Arg lacking Cys4057 and LDL was also essentially unaffected by high salt concentrations. This suggested the participation of a highly specific, salt-stable interaction between apo(a) and LDL in the formation of complexes that is independent of Cys-4047. We speculate that this interaction between apo(a) and apoB on LDL might bring the two -SH groups in such close proximity that a disulfide bond can be formed by oxidation. This covalent bond, finally, renders the complex stable to $\epsilon$-aminocaproic acid and detergents such as SDS.

\section{ACKNOWLEDGMENT}

We want to thank Dr. John McLean for apo(a) cDNA clones. We are also grateful to Margarete Fruhmann, Margit Stultschnig, and Harald Grillhofer for their technical assistance, Dr. G. Knipping for helpful discussions, and Dr. E. Zechner for editing the manuscript.

\section{REFERENCES}

Azrolan, N., Gavish, D. \& Breslow, J. L. (1991) J. Biol. Chem. 266, 13866-13872.

Boerwinkle, E., Leffert, C. C., Lin, J., Lackner, C., Chiesa, G. \& Hobbs, H. H. (1992) J. Clin. Invest. 90, 52-60.

Brunner, C., Kraft, H. G., Utermann, G. \& Müller, H. J. (1993) Proc. Natl. Acad. Sci. U.S.A. 90, 11643-11647.

Chiesa, G., Hobbs, H. H., Koschinsky, M. L., Lawn, R. M., Maika, S. D. \& Hammer, R. E. (1992) J. Biol. Chem. 267, 24369-24374.

Chung, B. Y., Wilkinson, T., Geer, J. \& Segrest, P. J. (1980) J. Lipid Res. 21, 284-291.

Cotten, M., Wagner, E., Zatloukal, K., Phillips, S., Curiel, D. T. \& Birnstiel, M. L. (1992) Proc. Natl. Acad.Sci.U.S.A.89, 6094-6098.

Deutscher, S. L. \& Hirschberg, C. B. (1986) J. Biol. Chem. 261, 96-100.

Deutscher, S. L., Nuwayhid, N., Stanley, P., Barak-Briles, E. I. \& Hirschberg, C. B. (1984) Cell 39, 295-299.

Dieplinger, H., Lackner, C., Kronenberg, F., Sandholzer, C., Lhotta, K., Hoppichler, F., Graf, H. \& König, P. (1993) $J$. Clin. Invest. 91, 397-401.

Edelberg, J. M., Pizzo, S. V. (1991) Fibrinolysis 5, 135-143.

Green, S., Issemann, I. \& Sheer, E. (1988) Nucleic Acids Res. $16,369$.

Griffiths, G., Quinn, P. \& Warren, G. (1983) J. Cell Biol. 96, 835-850.

Guevara, J., Jr., Spurlino, J., Jan, A. Y., Yank, C. Y., Tulinsky, A., Prasad, B. V. V., Gaubatz, J. V. \& Morrisett, J. D. (1993) Biophys. J. 64, 686-700.

Huby, T., Doucet, C., Dieplinger, H., Chapman, J., \& Thillet, J. (1994) Biochemistry 33, 3335-3341.

Ichinose, A. (1992) Biochemistry 31, 3113-3118.

Kamboh, M. I., Ferrell, R. E. \& Kottke, B. A. (1991) Am. J. Hum. Genet. 49, 1063-1074.
Koschinsky, M. L., Beisiegel, U., Henne-Bruns, D., Eaton, D. L. \& Lawn, R. L. (1990) Biochemistry 29, 640-644.

Koschinsky, M. L., Tomlinson, J. E., Zioncheck, T. F., Schwartz, K., Eaton, D. L. \& Lawn, R. M. (1991) Biochemistry 30, 5044-5051.

Koschinsky, M. L., Cote, G. P., Gabel, B. \& Van der Hoek, Y. Y. (1993) J. Biol. Chem. 26, 19819-19825.

Kostner, G. M. (1988) in Recent aspects of diagnosis and treatment of lipoprotein disorders, pp 255-263, Alan R. Liss Inc., New York.

Kostner, G. M. \& Grillhofer, H. (1991) J. Biol. Chem. 266, 21287-21292.

Kraft, H. G., Dieplinger, H., Hoye, E. \& Utermann, G. (1988) Arteriosclerosis 8, 212-216.

Kraft, H. G., Köchl, S., Menzel, H. J., Sandholzer, C. \& Utermann, G. (1992) Hum. Genet. 90, 2153-2161.

Krempler, F., Kostner, G. M., Bolzano, K., \& Sandhofer, F. (1979) Biochim. Biophys. Acta 575, 63-70.

Krempler, F., Kostner, G. M., Roscher, A., Haslauer, F., Bolzano, F. \& Sandhofer, F. (1983) J. Clin. Invest. 71, 1431-1441.

Lackner, C., Boerwinkle, E., Leffert, C. C., Rahmig, T. \& Hobbs, H. H. (1991) J. Clin. Invest. 87, 2153-2161.

Linton, M. F., Farese, R. F., Jr., Chiesa, G., Grass, D. S., Chin, P., Hammer, R. E., Hobbs, H. H., \& Young, S. G. (1993) J. Clin. Invest. 92, 3029-3037.

Malgaretti, N., Acquati, F., Magnaghi, P., Bruno, L., Pontoglio, M., Rocchi, M., Saccone, S., Della Valle, G., D'Urso, M., LePaslier, D., Ottolenghi, S. \& Taramelli, R. (1992) Proc. Natl. Acad. Sci. U.S.A. 89, 11584-11588.

McGregor, G. R. \& Caskey, C. T. (1989) Nucleic Acids Res. 17, 2365.

McLean, J. W., Tomlinson, J. E., Kuang, W. J., Eaton, D. L., Chen, E. Y., Fless, G. M., Scanu, A. M. \& Lawn, R. M. (1987) Nature 330, 132-137.

Menzel, H. J., Dieplinger, H., Lackner, C., Hoppichler, F., Lloyd, J. K., Müller, D. R., Labeur, C., Talmud, P. J. \& Utermann, G. (1990) J. Biol. Chem. 265, 981-986.

Phillips, M. L., Lembertas, A. V., Shumaker, V. N., Lawn, R. M., Shire, S. J. \& Zioncheck, T. F. (1993) Biochemistry 32, 3722-3728.

Rader, D. J., Cain, W., Zech, L. A., Usher, D., Brewer, Jr., H. B. (1993) J. Clin. Invest. 91, 443-447.

Sambrook, J., Fritsch, E. F. \& Maniatis, T. (1989) in Molecular cloning: A laboratory manual, 2nd ed., Cold Spring Harbor Laboratory Press, Cold Spring Harbor, NY.

Sandholzer, C., Feussner, G., Brunzell, J. D. \& Utermann, G. (1992) J. Clin. Invest. 90, 1958-1965.

Scanu, A. M. \& Fless, G. M. (1990) J. Clin. Invest. 85, 17091715.

Selden, R. F. (1987) in Current Protocols in Molecular Biology, pp 9.7.1-2, Greene Publishing Associates, New York.

Sommer, A., Gorges, R., Kostner, G. M., Paltauf, F. \& Hermetter, A. (1991) Biochemistry 30, 11245-11249.

Stanley, P., Narasimhan, S., Siminovitch, L. \& Schachter, H. (1975) Proc. Natl. Acad. Sci. U.S.A. 72, 3323-3327.

Trieu, V. N., Zioncheck, T. F., Lawn, R. M. \& McConathy, W. J. (1991) J. Biol. Chem. 266, 5480-5485.

Utermann, G. (1989) Science 246, 904-910.

Utermann, G. (1990) Curr. Opin. Lipidol. 1, 404-410.

Utermann, G., Menzel, H. J., Kraft, H. G., Duba, H. C., Kemmler, H. G. \& Seitz, C. (1987) J. Clin. Invest. 80, 458-465.

Wagner, E., Zenke, M., Cotten, M., Beug, H. \& Birnstiel, M. L. (1990) Proc. Natl. Acad. Sci. U.S.A. 87, 3410-3414.

White, A. L., Rainwater, D. L. \& Lanford, R. E. (1993) J. Lipid. Res. 34, 509-517.

Zatloukal, K., Wagner, E., Cotten, M., Phillips, S., Plank, C., Steinlein, P., Curiel, T. D. \& Birnstiel, M. L. (1992) Ann. N.Y. Acad. Sci. 660, 136-153.

Zechner, R., Dieplinger, H., Roscher, A. \& Kostner, G. M. (1984) Biochem. J. 224, 569-576. 